\title{
The Anatomy of Primary Care and Mental Health Clinician Communication: A Quality Improvement Case Study
}

\author{
Evelyn T. Chang, MD, MSHS ${ }^{1,2}$, Kenneth B. Wells, MD, MPH ${ }^{3,4}$, Alexander S. Young, MD, MSHS ${ }^{2,4,6}$, \\ Susan Stockdale, $P h D^{2}$, Megan D. Johnson, $M D, M P H^{2,5,6}$, Jacqueline J. Fickel, $P h D^{2}$, \\ Kevin Jou, $M D^{6}$, and Lisa V. Rubenstein, MD, MSPH
}

\begin{abstract}
'Department of Medicine, Veterans Affairs Greater Los Angeles Healthcare System, Los Angeles, CA, USA; ${ }^{2}$ VA Center for the Study of Healthcare Innovation, Implementation, and Policy, North Hills, CA, USA; ${ }^{3}$ Department of Health Policy \& Management, UCLA Fielding School of Public Health, Los Angeles, CA, USA; ' ${ }^{2}$ epartment of Psychiatry and Biobehavioral Sciences, UCLA School of Medicine, Los Angeles, CA, USA; ${ }^{5}$ RAND Corporation, Santa Monica, CA, USA; ${ }^{6}$ Department of Psychiatry, Veterans Affairs Greater Los Angeles Healthcare System, Los Angeles, CA, USA.
\end{abstract}

\begin{abstract}
BACKGROUND: The high prevalence of comorbid physical and mental illnesses among veterans is well known. Therefore, ensuring effective communication between primary care (PC) and mental health (MH) clinicians in the Veterans Affairs (VA) health care system is essential. The VA's Patient Aligned Care Teams (PACT) initiative has further raised awareness of the need for communication between PC and $\mathrm{MH}$. Improving such communication, however, has proven challenging.
\end{abstract}

OBJECTIVE: To qualitatively understand barriers to $\mathrm{PC}-\mathrm{MH}$ communication in an academic communitybased clinic by using continuous quality improvement (CQI) tools and then initiate a change strategy.

DESIGN, PARTICIPANTS, AND APPROACH: An interdisciplinary quality improvement (QI) work group composed of 11 on-site PC and $\mathrm{MH}$ providers, administrators, and researchers identified communication barriers and facilitators using fishbone diagrams and process flow maps. The work group then verified and provided context for the diagram and flow maps through medical record review (32 patients who received both $\mathrm{PC}$ and $\mathrm{MH}$ care), interviews (6 stakeholders), and reports from four previously completed focus groups. Based on these findings and a previous systematic review of interventions to improve interspecialty communication, the team initiated plans for improvement.

KEY RESULTS: Key communication barriers included lack of effective standardized communication processes, practice style differences, and inadequate PC training in MH. Clinicians often accessed advice or formal consultation based on pre-existing across-discipline personal relationships. The work group identified collocated collaborative care, joint care planning, and joint case conferences as feasible, evidence-based interventions for improving communication.

CONCLUSIONS: CQI tools enabled providers to systematically assess local communication barriers and facilitators and engaged stakeholders in developing possible solutions. A locally tailored CQI process focusing on

Published online April 9, 2014 communication helped initiate change strategies and ongoing improvement efforts.

KEY WORDS: primary care; mental health; communication; quality improvement; ambulatory care; primary care redesign.

J Gen Intern Med 29(Suppl 2):S598-606

DOI: $10.1007 / \mathrm{s} 11606-013-2731-7$

(c) Society of General Internal Medicine 2013

\section{INTRODUCTION}

Mental health $(\mathrm{MH})$ conditions are prevalent in primary care (PC) populations and can profoundly impact patients' overall health and well-being. ${ }^{1,2}$ Coordinating mental and physical health care for patients with both disorders optimizes health outcomes and reduces hospi-

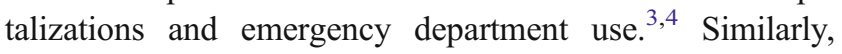
communication failures between $\mathrm{PC}$ and $\mathrm{MH}$ specialists are linked to worse outcomes. ${ }^{5-10}$ However, achieving communication between $\mathrm{MH}$ and $\mathrm{PC}$ is challenging. ${ }^{2,11-13}$ Fully coordinated $\mathrm{MH}$ and $\mathrm{PC}$ requires effective, timely communication between primary care providers (PCPs) and mental health providers (MHPs) about their shared patients. $^{5,14,15}$ In many health care settings, poor reimbursement for behavioral health care $e^{5,16-18}$ and limitations on sharing sensitive information between providers $5,18,19$ are barriers to communication.

In the Veterans Affairs (VA) health care system, where the high prevalence of comorbid physical and mental illnesses among veterans is well known, ${ }^{20-22}$ ensuring effective communication between $\mathrm{PC}$ and $\mathrm{MH}$ clinicians is essential. As a result, the VA has attempted to minimize these barriers through full parity between $\mathrm{MH}$ and medical benefits and inclusion of $\mathrm{MH}$ specialty notes in the electronic medical record (EMR). In addition, national VA directives mandate integrated care, ${ }^{23}$ and many $\mathrm{PC}$ practices include a collocated $\mathrm{MH}$ specialist, who provides $\mathrm{MH}$ care alongside primary care providers (PCPs) in the same clinic. $^{24}$ Yet, even in the VA, the goal of effective, timely communication between $\mathrm{PC}$ and $\mathrm{MH}$ remains elusive at local sites. ${ }^{21,25-28}$ Implementation of the VA's Patient 
Aligned Care Teams (PACT) $)^{29,30}$ in 2010 further raised awareness of the importance of interspecialty coordination and communication between $\mathrm{MH}$ and PC clinicians. Based on the Patient-Centered Medical Home (PCMH) model, PACT identifies care coordination between generalists and specialists, conceptualized as members of the PCMH Neighborhood, as a fundamental requirement. ${ }^{31,14}$

In this article, we present findings of a quality improvement (QI) iniative designed to qualitatively understand barriers to PC-MH communication in a VA academic community-based clinic and then initiate a change strategy. Despite an 8-year history of collocated psychiatrists in PC during the 1990s, this site has found $\mathrm{PC}-\mathrm{MH}$ coordination and communication challenging. ${ }^{32}$ A subsequent evaluation of the site's depression care $^{33}$ suggested that communication between $\mathrm{MH}$ and PC clinicians has remained limited. Recently under PACT, the site experienced renewed pressure to reduce $\mathrm{MH}$ backlogs for patients referred from PC. Site management proposed to shift responsibility for managing patients with stable, chronic $\mathrm{MH}$ issues from MH back to PC. This plan was opposed by PC and $\mathrm{MH}$ providers and also revealed underdeveloped PC-MH communication. As a result, the site leadership abandoned the transfer project in favor of more fundamental efforts to first improve communication and coordination, thus providing the motivation for this project.

Our local QI project was carried out within the context of a larger regional evidence-based quality improvement $(\mathrm{EBQI})^{34}$ initiative. EBQI is an approach that emphasizes continuous quality improvement (CQI) methods, accompanied by the explicit use of theory, evidence from the literature, and measurement, within a researcher/clinical partnership. By describing the project, we hope to provide other health care organizations and clinicians struggling with similar challenges with a framework they can use for improving local PC-MH communication.

\section{METHODS}

Overview. We used CQI diagnostic methods as thought tools for investigating local communication problems and engaging stakeholders in problem solving. We used relational coordination, defined as "a mutually reinforcing process of interaction between communication and relationships carried out for the purpose of task integration," as a framework for our investigation. $^{35}$ The theory postulates that effective communication, shared goals and knowledge, and mutual respect within organizations link tightly to quality and efficiency. ${ }^{35-37}$ We collected relevant local data on communication patterns and barriers. Then we used the results of a systematic review of PC-MH communication ${ }^{7}$ and local data to develop and initiate strategies for improvement.
As noted above, this QI effort initiated by the authors (ETC, LVR) was based on local leadership concerns about PC-MH communication. The project was supported through the VA Assessment and Improvement Laboratory (VAIL), one of five regional VA PACT Demonstration Laboratories. $^{34,38-40}$ The site's PACT Quality Council, an interdisciplinary group of local clinical and administrative leaders, approved the project goals and activities in November 2011. Institutional Review Board approval was not required for this nonresearch operations project. ${ }^{41}$

Setting. The project was conducted in a multispecialty academic community-based outpatient clinic serving over 16,000 veterans in Southern California. The site includes 23 full- or part-time PCPs who provide continuity of PC for veterans; 36 psychologists, psychiatrists, and social workers; and $\mathrm{MH}$ and $\mathrm{PC}$ residents. On-site comprehensive outpatient MH services (i.e., individual or group therapy, psychopharmacology) and chemical dependency services are located in a separate building from the main PC building.

Participants. We presented the problem of PC-MH communication during a regional QI coaching session to nine VAIL participants from three sites who were familiar with PC-MH integration issues. We used findings from this initial brainstorming session to prepare for recruiting and initiating a local QI work group consisting of 11 front-line clinicians and key stakeholders from the local site. The work group included three psychiatrists, five PCPs, one PC nurse care manager, one researcher, and an information technology specialist. A clinician-researcher trained in both health services research and QI facilitated and collected data for the project (ETC).

Meetings. The regional QI coaching session was 1.5 hours. The local QI work group held four monthly 1-hour meetings from December 2011-March 2012 to achieve consensus on key barriers and recommendations. Intervening work group member "homework" included reviewing relevant data or answering relevant survey questions. For members who could not attend group meetings, the facilitator conducted individual or small group interviews on key points of discussion.

Mixed methods CQI diagnostic approach. We used a fishbone diagram and process flow charts to identify possible barriers to PC-MH communication. We then used focus groups, semistructured interviews, chart reviews, and provider surveys to further explore and validate the branches of the fishbone. The facilitator collected data and reviewed results with the work group. The work group used the final fishbone diagram and flow charts that integrated the additional exploration and validation steps as the basis for considering strategies for improvement. 
During the regional QI coaching session, the facilitator used an Ishikawa fishbone diagram approach similar to root-cause analysis ${ }^{42,43}$ to identify initial potential barriers to PC-MH communication. The local QI work group then revised the initial draft diagram through additional group brainstorming. Key branches of the fishbone diagram resulting from this two-step process were: "Process," "Communication Tools," "Provider characteristics," "Non-VA providers," and "Culture."

To verify that all major categories of communication issues had been identified, the facilitator reviewed reports from four focus groups conducted by the broader EBQI PC$\mathrm{MH}$ integration initiative at the local site and abstracted information relevant to communication. One group consisted of patients, one of psychiatrists, one of PCPs, and one of social workers, each with 5-8 participants, conducted between February and April 2011.

To further assess "Process" and "Communication Tools," the facilitator developed process flow charts for PC contacting $\mathrm{MH}$ and for $\mathrm{MH}$ contacting $\mathrm{PC}$ by interviewing each work group clinician individually. Interview questions asked for usual methods of contact for routine and emergency problems and for steps taken if the provider was not available. The facilitator aggregated the individual responses for each discipline and reviewed the results with the work group for confirmation. The facilitator then conducted semistructured interviews with current site leaders (an $\mathrm{MH}$ and a PC leader) to review and validate the process flow charts.

To investigate the problems related to "Culture" and their historical roots at the site, the facilitator conducted semistructured interviews with two of three formerly collocated psychiatrists and two PCPs who had practiced at the site during the early 1990s. To investigate "Communication Tools," the facilitator conducted a chart review to assess EMR-based PC-MH communication. The review included 32 randomly selected medical records from among 279 veterans with an established PCP from the work group who had an encounter with a psychologist or psychiatrist during November 2011. The record abstraction recorded any documentation of PCP and MHP communication, including acknowledgement of the other discipline's care plans. Finally, to further verify the fishbone in terms of "Communication Tools," "Process," "Provider characteristics," and "Culture" isues, the facilitator conducted an anonynous provider survey of all front-line $\mathrm{PC}$ and $\mathrm{MH}$ clinicians at the site.

Strategies for improvement. To identify candidate strategies, the work group reviewed interventions for improving communication identified through a prior systematic review of PC-MH communication. ${ }^{7}$ Work group members then suggested additional strategies based on barriers listed in the final fishbone and flow charts. Then they completed an anonymous online survey to vote for the most feasible interventions. The facilitator further assessed feasibility by reviewing highly ranked interventions with an advisory group comprised of a psychologist, social workers, and a geriatrician from the site. Interventions deemed by the work group and advisory group to be the most feasible were to undergo Plan-Do-Study-Act (PDSA) cycles for further development.

\section{RESULTS}

In Fig. 1, we present the final fishbone diagram summarizing the core result of the QI work group's diagnostic efforts as derived from group brainstorming and additional exploratory activities. In Table 1, we summarize our main results, indicating primary sources of information for that finding (flow charts, interviews, chart review, and provider survey) and links to the fishbone diagram.

Process. Both PCPs and MHPs stated that, despite having a shared EMR, the single biggest barrier to communication was identifying which providers (i.e., PCP, primary MHP) belonged on a patient's care team. They also agreed that identifying the attending to contact if a resident provided continuity of care or if the primary provider was unavailable was equally challenging. PCPs cited logistical difficulties in contacting psychiatry residents for shared patients; resident supervision could vary weekly, and residents did not have VA e-mail addresses or known office phone numbers. Given recent PACT implementation, psychiatrists often did not know which teamlet member to contact if the PCP was unavailable. PCPs also noted challenges identifying a responsible MHP if different types of MH specialists were involved in a patient's care, including clinicians providing individual or group therapy, psychopharmacology, or substance abuse services. Neither PCPs nor MH specialists thought they could reliably contact the other service for emergencies, despite the existence of emergency protocols.

The process flow charts shown in Figs. 2 and 3 illustrate that most providers used unofficial workarounds rather than broadly understood and reliable communication methods. In emergencies, PCPs first paged the Consult-Liaison (CL) service per protocol. However, CL was often unavailable; one PCP stated that he was "unable to reach a MH provider when paged by beeper and even sometimes overhead pages." Other PCPs called the clerk or the nurse in the outpatient MH building to leave messages for the psychiatrists. Others looked for available psychiatrists in medical specialty clinics in the main ambulatory building. In one instance, a PCP called the nearby VA hospital emergency room to curbside, or seek advice from, a psychiatrist. 


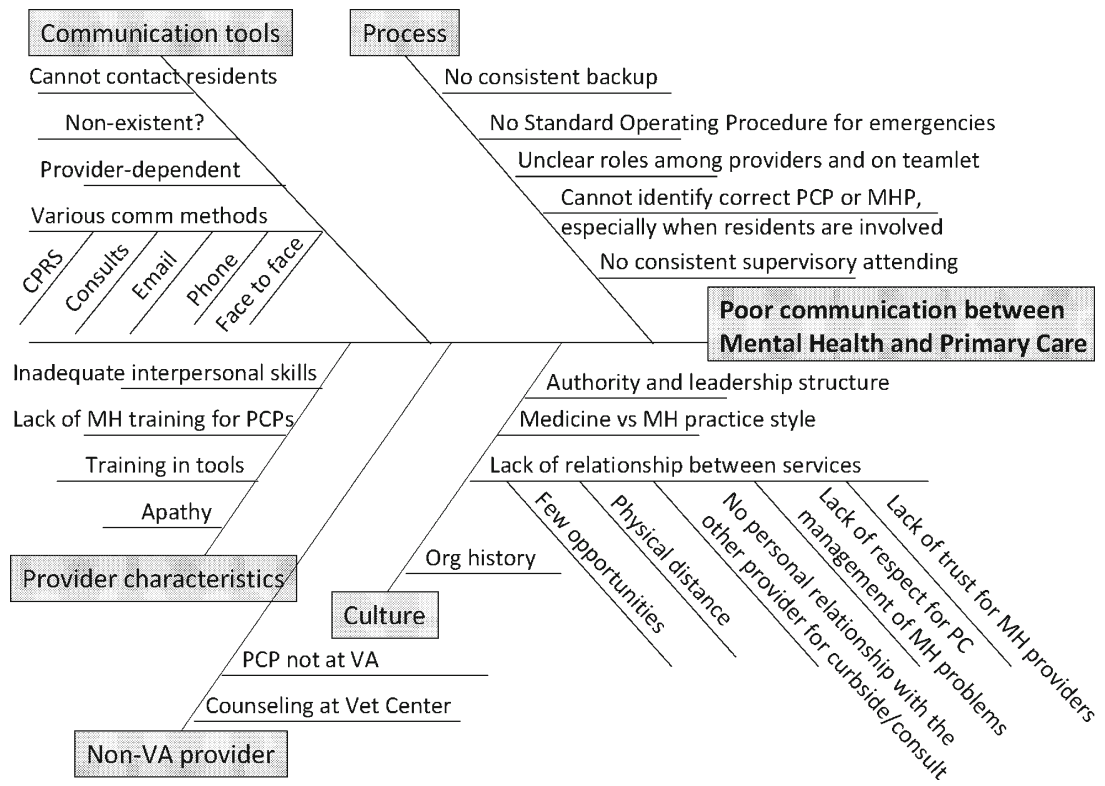

Figure 1. Fishbone diagram for contributors to poor communication between primary care providers (PCP) and mental health providers (MHP). $M H$ mental health, $M H P$ mental health provider, $P C$ primary care; $P C P$ primary care provider.

Table 1. Supporting Evidence for Fishbone Diagram Using Further Diagnostic Activities

\begin{tabular}{|c|c|c|}
\hline Data source & Link to fishbone diagram & Result \\
\hline $\begin{array}{l}\text { Review of reports from prior } \\
\text { local focus groups conducted } \\
\text { to explore PC-MH integration } \\
\text { problems }\end{array}$ & $\begin{array}{l}\text { Communication Tools, Process, Provider } \\
\text { Characteristics and Culture: Verified } \\
\text { completeness of the fishbone categories } \\
\text { by abstracting information relevant to } \\
\text { communication }\end{array}$ & $\begin{array}{l}\text { - Providers and patients were frustrated with the wait time for new } \\
\text { MH consultations. } \\
\text { - PCPs were dissatisfied with lack of an overall MH treatment plan } \\
\text { and lack of care coordination. } \\
\text { - Psychiatrists thought that PCPs should provide more } \\
\text { psychopharmacologic care and that some consultations were } \\
\text { poorly written }\end{array}$ \\
\hline $\begin{array}{l}\text { Semistructured interviews } \\
\text { with current site leaders in } \\
\text { MH and PC }\end{array}$ & $\begin{array}{l}\text { Communication Tools, Process: Verified } \\
\text { and expanded on flow chart findings }\end{array}$ & $\begin{array}{l}\text { - MH care at the site was provided in several ways: PCPs could } \\
\text { manage uncomplicated depression through an evidence-based } \\
\text { depression nurse care management program. }{ }^{38} \text { For emergent } \\
\text { consultations, a Consult-Liaison service was available by pager } \\
\text { on weekdays during business hours. Collocated psychiatrists } \\
\text { were available only for specialty clinics: women's health, recent } \\
\text { service veterans, and geriatrics. } \\
\text { - Psychiatry trainees provided continuity of care in } 80 \% \text { of cases }\end{array}$ \\
\hline $\begin{array}{l}\text { Semistructured interviews } \\
\text { with previously collocated } \\
\text { psychiatrists and internists }\end{array}$ & $\begin{array}{l}\text { Culture: Explored local organizational } \\
\text { history }\end{array}$ & $\begin{array}{l}\text { - Collocation did not seem to change MH treatment rates, }{ }^{33} \text { resulting } \\
\text { in backlogged cases for specialty MH. PC did not consider } \\
\text { collocated psychiatrists essential enough to financially support them } \\
\text { with PC-funded positions, ending } 8 \text { years of collocation. } \\
\text { - Depression care management program weakly supported by MH } \\
\text { and PC leadership. } \\
\text { - Subsequent attempts to re-collocate MHPs into PC met resistance }\end{array}$ \\
\hline Chart review & $\begin{array}{l}\text { Communication Tools: Verified problems } \\
\text { with and common strategies using the } \\
\text { EMR }\end{array}$ & $\begin{array}{l}\text { - Chart review confirmed the between-provider variation in } \\
\text { documented communication strategies and acknowledgement of a } \\
\text { patient's physical or MH problems. PCPs acknowledged MH } \\
\text { problems in } 16 / 32 \text { charts, psychiatrists acknowledged physical } \\
\text { problems in } 9 / 32 \text { charts, and psychologists and social workers } \\
\text { acknowledged physical health problems in } 0 / 32 \text { charts. Identified } \\
\text { communication strategies included adding the other provider as a } \\
\text { co-signer, referencing previous notes, and writing short messages } \\
\text { within the clinic note directly to a provider }\end{array}$ \\
\hline Site $\mathrm{PC}$ and $\mathrm{MH}$ survey & $\begin{array}{l}\text { Communication Process, Tools, Provider } \\
\text { Characteristics and Culture: Verified } \\
\text { problems }\end{array}$ & $\begin{array}{l}\text { - Response rate was } 39 \% \text { for } 23 \text { PCPs }(n=9) \text { and } 17 \% \text { for } 36 \\
\text { psychiatrists, psychologists, and social workers }(n=6) \text {. } \\
\text { - The survey confirmed the major communication barriers for the } \\
\text { site. Both PCPs and MHPs agreed that they could not easily } \\
\text { identify the provider primarily responsible for a patient's physical } \\
\text { or MH care, the attending to contact if the primary provider was a } \\
\text { trainee, and the backup provider. PCPs felt that MHPs were } \\
\text { inaccessible during MH emergencies, and MHPs felt that PCPs } \\
\text { lacked familiarity with MH treatments. } \\
\text { - Mutual respect }\end{array}$ \\
\hline
\end{tabular}




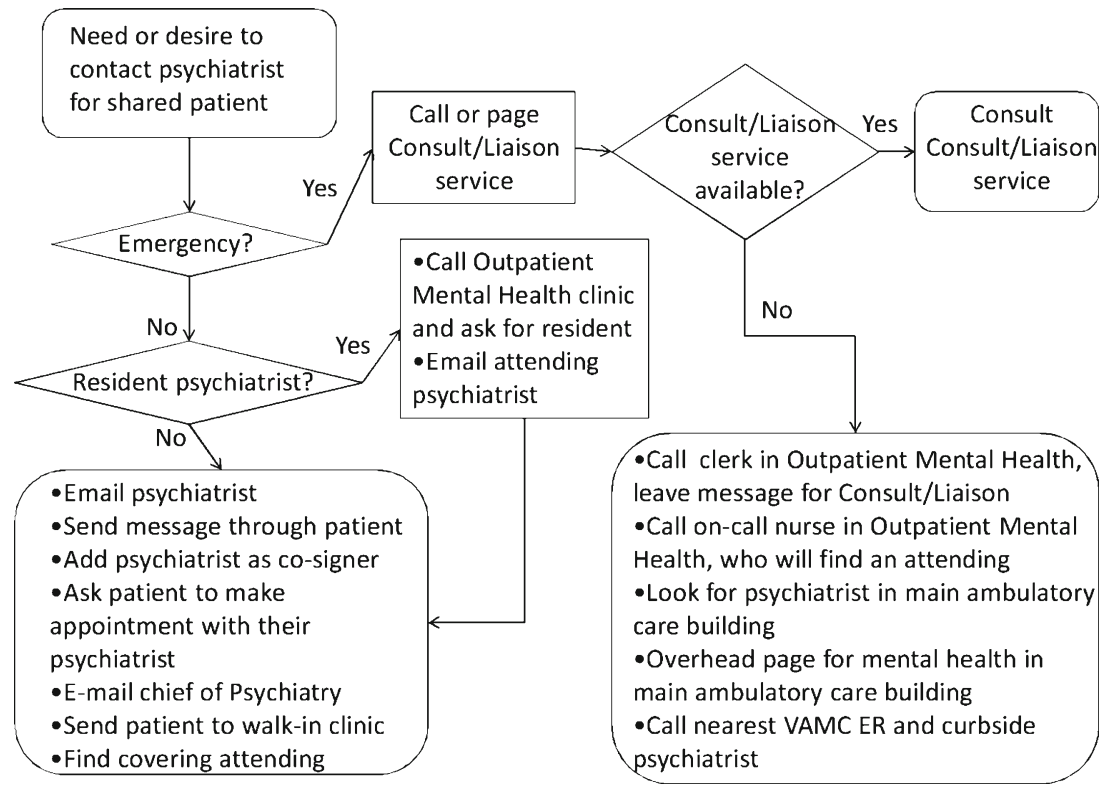

Figure 2. Flow chart of how primary care providers (PCPs) contact psychiatrists. ER emergency room, VAMC VA medical center.

Furthermore, patients were often cared for by resident psychiatrists. PCPs tried various strategies to contact the resident or the attending that had co-signed the most recent progress note, including calling the outpatient MH building for the resident, emailing the attending, adding a psychiatrist as a co-signer to the PC progress note, sending a message through the patient, or asking the patient to make an appointment with their MHP. Chart review confirmed between-provider variation in documented communication strategies.
Communication tools. Some providers thought that there were too many communication methods (e.g., phone, email, face-to-face, EMR) to use without knowing provider preferences for their use. Face-to-face or phone interactions were perceived as time consuming and seldom used. Typically, MH and PCPs relied upon EMR notes as their main communication tool, sometimes using the co-signing feature to flag notes for providers from another discipline. However, providers agreed that co-signatures seemed inadequate for addressing a routine question or problem,

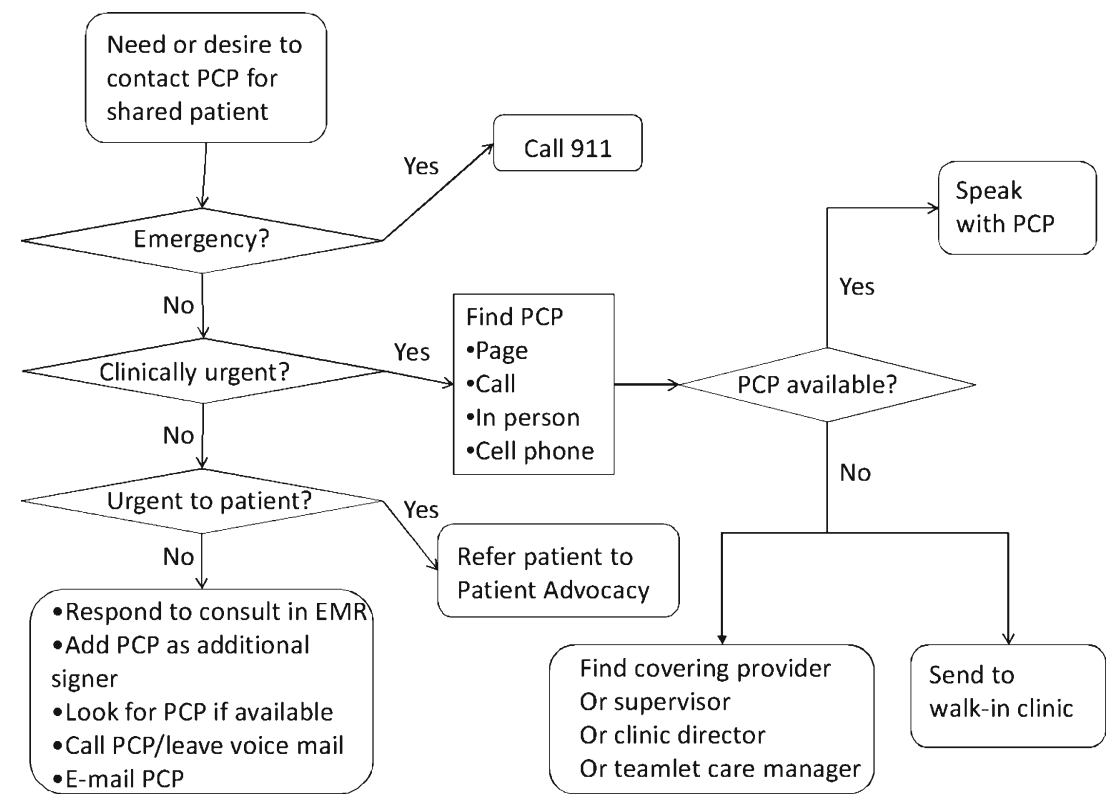

Figure 3. Flow chart of how psychiatrists contact primary care providers (PCPs). EMR electronic medical record, PCP primary care provider. 
which was subsequently confirmed upon chart review. In one chart, the PCP had directed a routine question about a tentative medication change to the MHP in two progress notes during a 6-month period; the psychiatrist had cosigned both PCP notes without acknowledging the question or changing the treatment plan.

Provider characteristics. MHPs believed that PCPs were not sufficiently trained to diagnose and manage $\mathrm{MH}$ conditions. They thought PCPs lacked familiarity with basic $\mathrm{MH}$ therapies, particularly psychotropics: "PCPs have indicated an aversion to prescribing any psychiatric medications to psychiatric patients, even if they routinely prescribe these medications for other problems." Given PC discomfort with $\mathrm{MH}$, psychiatrists in the work group also did not consider many emergency consultations from PCPs to be "true" emergencies; many PC-generated consultations also seemed "without just cause." Similarly, half of the MH survey respondents thought PCPs could not accurately assess patients with mental illnesses. PCP respondents, on the other hand, perceived that MHPs did not respect or value PCPs as partners in caring for shared patients with mental illnesses.

Culture. Providers also noted an underlying cultural divide among PC and MH. Differences in practice styles made communication difficult, particularly with certain types of MH specialists. Upon chart review, we found that while psychiatrists occasionally acknowledged physical health problems within progress notes, psychologists and social workers rarely did. Moreover, PCPs found it difficult to extract significant treatment goals or plans from $\mathrm{MH}$ progress notes. The focus group PCPs remarked that "there are mental health treatment notes, but no overall treatment plan" in the EMR. If a MH care plan existed, PCPs thought it seemed "generic and not patient-specific." Similarly, PCPs generally were perceived to have a limited understanding of $\mathrm{MH}$ concepts and goals. One surveyed MHP stated, "When I have attempted to talk with [physicians], most are confused what I'm even attempting to achieve." Furthermore, providers observed that few opportunities existed for interactions between the disciplines. Another surveyed MHP commented, "The implication is that there is already contact, which there rarely is." Both $\mathrm{PC}$ and $\mathrm{MH}$ agreed that personal acrossdiscipline relationships facilitated curbside and formal consultations for their patients despite the geographic separation. Such relationships, however, were primarily among attendings, rather than with the $\mathrm{MH}$ trainees who provided continuity of care for most MH patients.

Interviews with psychiatrists and PCPs who had practiced at the site during the early 1990s further revealed that these cultural barriers have persisted despite multiple efforts in PC-MH integration. When the PC clinic included collocated psychiatrists, the interviewed providers felt that they functioned well as an interdisciplinary team. However, MH illnesses were not managed on a population level, and $\mathrm{MH}$ screening and treatment rates did not seem to improve within $\mathrm{PC},{ }^{33}$ resulting in backlogged specialty $\mathrm{MH}$ referrals. Because of access problems to specialty $\mathrm{MH}$, the collocated psychiatrists were shifted into specialty $\mathrm{MH}$. The site subsequently implemented a depression care management program that did not achieve bilateral $\mathrm{MH}$ and $\mathrm{PC}$ leadership support and was ultimately only marginally sustained. Further attempts to replace MHPs in PC to comply with the national VA mandate ${ }^{23}$ for PC-MH integration were met with resistance from the local leadership.

Non-VA providers. The work group also identified communication with non-VA health care system providers, including the local Veteran Center, which provides marriage and family counseling for veterans, as an important issue. This site did not have standardized processes to contact or share medical records with such non-VA providers.

Strategies for improvement. The strategies used by studies with positive outcomes in the prior systematic review ${ }^{7}$ included: an integrated treatment plan that combines both physical and $\mathrm{MH}$ goals, multidisciplinary team meetings, regular joint case conferences, joint patient consultation with PC and MHP, a case manager for high utilizers, and medication therapy management by pharmacists. Anonymous online voting process and in-depth interviews with advisory group members identified joint treatment planning and joint case conferences as immediately feasible interventions and collocated collaborative care ${ }^{44,45}$ as feasible over time.

Published interventions did not address all issues raised during the diagnostic process. Table 2 shows the additional suggested strategies for improvement that the work group considered to be feasible.

The work group then began PDSA cycles for joint care planning to identify a patient's interdisciplinary care team members (i.e., PCP, primary MHP), specify backup providers, and establish care plans using a team approach. Concurrently, the work group began to develop joint case conferences to facilitate cross-discipline education and foster relationships across disciplines. During the study, with the support of local leadership, the site collocated a team of MH specialists within PC. An interdisciplinary research/clinical team assisted with implementation of these initiatives.

\section{DISCUSSION}

In this case study, we used CQI methods to diagnose communication problems between $\mathrm{MH}$ and $\mathrm{PC}$ at one ambulatory care site and identified possible locally accept- 
Table 2. Major Barriers to Communication between Primary Care Providers (PCP) and Mental Health Providers (MHP) and Possible Solutions Identified by the Quality Improvement (QI) Work Group

\begin{tabular}{|c|c|}
\hline Fishbone diagram branch: major barriers & Possible solutions \\
\hline $\begin{array}{l}\text { Process: Cannot identify correct MHP or PCP, } \\
\text { especially when trainees are involved }\end{array}$ & $\begin{array}{l}\text { (1) Designate a primary MHP for each patient in the EMR, parallel to the designation } \\
\text { of a PCP } \\
\text { (2) Identify a single supervisor of record for each trainee and make the information } \\
\text { available electronically } \\
\text { (3) Disseminate contact information for MH trainees who serve as primary MHPs } \\
\text { electronically }\end{array}$ \\
\hline $\begin{array}{l}\text { Process: Cannot identify a backup } \mathrm{MH} \text { or PC } \\
\text { provider }\end{array}$ & $\begin{array}{l}\text { (1) Designate collocated MHP in PC to serve as backup when the primary MHP cannot } \\
\text { be reached for urgent issues } \\
\text { (2) Designate the patient's PACT team as the backup for medical emergencies } \\
\text { (3) Electronically identify a trusted MHP as backup to the primary MHP for especially } \\
\text { complex patients and similarly identify a backup PCP }\end{array}$ \\
\hline $\begin{array}{l}\text { Process: Cannot identify who to contact in } \\
\text { MH or medical emergencies } \\
\text { Provider Characteristics and Culture: Create } \\
\text { a relationship between services and promote } \\
\text { improved PC knowledge of MH }\end{array}$ & $\begin{array}{l}\text { (1) Designate an on-call psychiatrist or psychologist, perhaps a collocated MHP } \\
\text { (2) Develop protocols during emergencies to transfer patient off site when necessary } \\
\text { (1) Adopt a collocated collaborative care model } \\
\text { (2) Hold joint case conferences } \\
\text { (3) Offer brief, targeted training sessions } \\
\text { (4) Include MHP during regular PACT meetings } \\
\text { (5) Use e-Consultations in EMR to educate PCPs } \\
\text { (6) Offer webinars } \\
\text { (7) Include MHP during PACT daily huddles }\end{array}$ \\
\hline
\end{tabular}

MH mental health, MHP mental health provider, EMR electronic medical record, VAMC VA Medical Center, PC primary care, PCP primary care provider, ER emergency room, PACT Patient Aligned Care Team

able solutions. Our work extends the previous research on barriers in PC-MH communication by providing a systematic "on-the-ground" assessment of a site as a foundation for improvement. Our analysis revealed the importance of local logistical barriers not previously addressed in most literature, such as difficulty identifying who to contact when multiple MHPs or PC staff participate in patient care. Identification of such barriers, a key component of many successful CQI initiatives, can provide a basis for system redesign. ${ }^{46}$

Among the key elements of relational coordination, this project identified ineffective communication processes, lack of shared goals and knowledge, and lack of mutual respect as barriers at this site. The lack of standardized communication processes led to provider workarounds and inefficient workflows. Standardizing communication processes to facilitate active and consistent communication may be key to improving quality of $\mathrm{MH}$ care in PC settings. Practice style differences between medical and $\mathrm{MH}$ providers ${ }^{9,47-51}$ created further communication difficulties, such as the difficulties in interpreting the other discipline's notes and variability in addressing mental or physical health problems in notes. Further research on making EMR notes more meaningful across $\mathrm{MH}$ and $\mathrm{PC}$ is essential, since both specialties preferred the EMR as the primary mode of communication. ${ }^{47}$ Lack of mutual respect was particularly evident in $\mathrm{MH}$ specialist attitudes toward PCPs, whom they perceived as having inadequate knowledge and training related to $\mathrm{MH}$ conditions. PCPs agreed they had deficits in this area but also indicated that their roles in caring for $\mathrm{MH}$ conditions were underappreciated by MHPs. We expect that caring for patients together as an interdisciplinary team will improve mutual respect.

Several of the communication barriers identified by the work group have since been addressed. The work group tested a template for joint treatment planning and held a well-attended joint Medicine/Psychiatry Grand Rounds. With changes in leadership and the guidance of the VAIL interdisciplinary research/clinical team, the site has successfully collocated $\mathrm{MH}$ specialists to PC to provide new consults for non-urgent and emergent cases and group therapy. The site also began identifying individuals to serve as treatment coordinators for each $\mathrm{MH}$ patient, which was accelerated by a new national VA mandate that the patient's "Mental Health Treatment Coordinator" be clearly designated in the EMR.

The case study reported here is exploratory. First, the barriers reported here may not be generalizable. Most VA community-based clinics are smaller, non-academic, and employ fewer MH staff. $^{24,52}$ In addition, VA sites have adopted varying models for PC-MH integration, which has resulted in tremendous variation in local pathways and barriers. ${ }^{24,28,53,54}$ Other VA sites, however, have reported similar barriers, including cultural barriers ${ }^{26,28}$ and the need for PCP training in $\mathrm{MH}^{28}$ The national mandate to identify MH Treatment Coordinators additionally validates the fishbone branch related to process as a national issue. Second, while we used relational coordination theory as a framework, this study was not designed to test the theory. Third, the work group included more PCPs than MHPs and may have over-represented PCP views. Finally, this QI initiative remains a work in progress at the time of this report. It will not be possible to separate this work 
group's efforts from related and ongoing QI at the site; summative evaluation of outcomes for patients with medical and $\mathrm{MH}$ comorbidities at the site level, however, is underway.

While we expect that barriers, pathways, and potential solutions will differ across local settings, the CQI approach ${ }^{43}$ described in this article can be applied to any site. The resources and data collection methods used here are within the reach of typical local QI efforts and illustrate several ways of triangulating, or validating, QI participant feedback with actual data collection. We hope that similar local CQI-based problem-solving can provide a way forward for other sites aiming to improve $\mathrm{PC}-\mathrm{MH}$ communication.

In summary, achieving seamless PC-MH communication in local VA sites is challenging, even in the presence of system-level mandates and resources. Understanding the anatomy of a local site's communication problems may help bridge the gap between PC-MH communication goals and local realities. Our work can serve as a starting point for both VA and non-VA sites in considering how to advance coordinated PC-MH care.

Acknowledgments: We would like to thank the interdisciplinary work group members (Kevin Jou, MD, Jackie Fickel, PhD, Megan Johnson, MD, MPH, Maria Davis, NP, Maria Zambrano, NP, Mai Pham, MD, Sue Donovan, RN, Wendell Ching, MD, Lisa Rubenstein, $M D, M S P H$, Susan Vivell, PhD, MBA, Randy Mervis, MD), the advisory group (Cathy Alessi, MD, Dana Melching, LCSW, Marti Waite, LCSW, Sarah Minden Weil, LCSW, Ed Chaney, PhD), Eloy DeJesus, NP, the VAIL QI leaders, and the site administrators (Lisa Altman, MD, Steve Ganzell, PhD, Ali Kazim, MD, Allen Suh, MPH) for their help with this project.

We would like to thank Pamela Diefenbach, MD, Maga TricheJackson, MD, MSHS, Wendell Ching, MD, and Lisa Rubenstein, MD, MSPH, for interviews about the site's organizational history.

We would also like to thank John Ovreveit, PhD, and the VAIL committee for reviewing earlier drafts of the manuscript.

The views expressed in this article are those of the authors and do not necessarily reflect the position or policy of the Department of Veterans Affairs, or the US government, or other affiliated institutions.

Funders: This work was undertaken as part of the Veterans Administration's PACT Demonstration Laboratory initiative, supporting and evaluating VA's transition to a patient-centered medical home. Funding for the PACT Demonstration Laboratory initiative is provided by the VA Office of Patient Care Services.

Funding support provided by VA Office of Academic Affiliations, Health Services Research and Development through the Health Services Fellowship Training Program (TMP 65-020).

Prior presentations: Podium presentation summarizing these findings was presented at Academy Health Annual Research Meeting on June 23-25, 2013, in Baltimore, $M D$.

A poster presentation summarizing these findings was presented at the Society of General Internal Medicine 36th Annual Meeting on April 25, 2012, in Denver, CO.

A web-based seminar summarizing these finding was presented during the VA Assessment and Improvement Laboratory PACT/ Mental Health Cyberseminar on October 17, 2012.

Conflict of Interest: The authors declare that they do not have a conflict of interest.
Corresponding Author: Evelyn T. Chang, MD, MSHS; Department of GIM (111G), VA Greater Los Angeles Healthcare System, 11301 Wilshire Blvd, Los Angeles, CA 90073, USA (e-mail: evelyn.chang@va.gov).

\section{REFERENCES}

1. Kessler R, Chiu W, Demler O, Walters E. Prevalence, severity, and comorbidity of twelve-month DSM-IV disorders in the National Comorbidity Survey Replication (NCS-R). Archives of General Psychiatry. 2005;62(6):617-627.

2. Wang PS, Berglund P, Kessler RC. Recent care of common mental disorders in the United States : prevalence and conformance with evidence-based recommendations. J Gen Intern Med. 2000;15(5):284292.

3. Yoon J, Yano E, Altman L, et al. Reducing costs of acute care for ambulatory case-sensitive medical conditions: the central roles of comorbid mental illness. Med Care. 2012.

4. Unutzer J, Katon WJ, Fan M-Y, et al. Long-term cost effects of collaborative care for late-life depression. Am J Manag Care. 2008; 14:95-100.

5. Butler M, Kane RL, McAlpine D, et al. Integration of Mental Health/ Substance Abuse and Primary Care No. 173. Rockville: Agency for Healthcare Research and Quality; 2008.

6. Stange $\mathbf{K}$. Shared care: what mix of generalist and specialist care optimizes patient outcomes? J Family Practice. 1999;48(3):177-179.

7. Foy R, Hempel S, Rubenstein L, et al. Meta-analysis: the effect of interactive communication between collaborating primary care physicians and specialists. Ann Intern Med. 2010;152(4):247-258.

8. Kilbourne AM, Greenwald DE, Bauer MS, Charns MP, Yano EM. Mental Health Provider Perspectives Regarding Integrated Medical Care for Patients with Serious Mental Illness. Adm Policy Ment Health. Jul 7 2011.

9. Knowles P. Collaborative communication between psychologists and primary care providers. J Clin Psychol Med settings. 2009;16(1):72-76.

10. Durbin J, Barnsley J, Finlayson B, et al. Quality of communication between primary health care and mental health care: An examination of referral and discharge letters. J Behav Health Serv Res. Aug 2012.

11. Liu CF, Campbell DG, Chaney EF, Li YF, McDonell M, Fihn SD. Depression diagnosis and antidepressant treatment among depressed VA primary care patients. Administration and policy in mental health. 2006;33(3):331-341.

12. Henke RM, Chou AF, Chanin JC, Zides AB, Scholle SH. Physician attitude toward depression care interventions: implications for implementation of quality improvement initiatives. Implement Sci. 2008;3:40.

13. Young AS, Klap R, Sherbourne CD, Wells KB. The quality of care for depressive and anxiety disorders in the United States. Arch Gen Psychiatry. 2001;58(1):55-61.

14. Taylor E, Lake T, Nysenbaum J, Peterson G, Meyers D. Coordinating care in the medical neighborhood: critical components and available mechanisms. Rockville: Agency for Healthcare Research and Quality; 2011.

15. Alakeson V, Frank RG, Katz RE. Specialty Care Medical Homes for People with Severe. Persistent Mental Disorders. Health Affairs. 2010;29(5):867-873.

16. Nutting $\mathbf{P}$, Gallagher $\mathbf{K}$, Riley $\mathbf{K}$, et al. Care management for depression in primary care practice: Findings from the RESPECT-Depression Trial. Ann Fam Med. 2008;6:30-37.

17. Henke R, McGuire T, Zaslavsky A, Ford D, Meredity L, Arbelaez J. Clinician- and organization-level factors in the adoption of evidencebased care for depression in primary care. Health Care Manage Rev. 2008;33(4):289-299.

18. Kilbourne AM, Schulberg HC, Post EP, Rollman BL, Belnap BH, Pincus HA. Translating evidence-based depression management services to community-based primary care practices. Milbank $\mathrm{Q}$. 2004;82(4):631-659.

19. Institute of Medicine US Committee on Crossing the Quality Chasm: Adaptation to Mental Health and Addictive Disorders. Improving the Quality of Health Care for Mental and Substance-Use Conditions. Washington: National Academies Press; 2006. 
20. Jordan B, Schlenger WE, Hough R, et al. Lifetime and current prevalence of specific psychiatric disorders among Vietnam veterans and controls. Archives of General Psychiatry. 1991;48(3):207-215.

21. Yano E, Chaney E, Campbell D, et al. Yield of practice-based depression screenings in VA primary care settings. J Gen Intern Med. 2012;27(3):331-338.

22. Seal KH, Bertenthal D, Miner CR, Sen S, Marmar C. Bringing the war back home: Mental health disorders among 103,788 US veterans returning from Iraq and Afghanistan seen at Department of Veterans Affairs facilities. Archives of Internal Medicine. 2007;167(5):476-482.

23. VHA Handbook 1160.01 Uniform Mental Health Services in VA Medical Centers and Clinics. Washington, DC: Department of Veterans Affairs: Office of Patient Care Services; 2008.

24. Chang ET, Rose DE, Yano EM, et al. Determinants of readiness for primary care-mental health integration (PC-MHI) in the VA health care system. J Gen Intern Med. 2013;28(3):353-362.

25. Tai-Seale M, Kunik M, Shepherd A, Kirchner J, Gottumukkala A. A case study of early experience with implementation of collaborative care in the Veterans Health Administration. Population Health Management. 2010;13(6):331-337.

26. Fickel J, Parker L, Yano E, Kirchner J. Primary care-mental health collaboration: an example of assessing usual practice and potential barriers. J Interprofessional Care. 2007;21(2):207-216.

27. Kirchner J, Cody M, Thrush C, Sullivan G, Rapp CG. Identifying factors critical to implementation of integrated mental health services in rural VA community-based outpatient clinics. Journal of Behavioral Health Services and Research. 2004;31(1):13-25.

28. Benzer JK, Beehler S, Miller C, et al. Grounded theory of barriers and facilitators to mandated implementation of mental health care in the primary care setting. Depress Res Treat. 2012;2012:597157.

29. The Advanced Medical Home: A Patient-Centered, Physician-Guided Model of Health Care: Policy Monograph of the American College of Physicians;2006.

30. Joint Principles of the Patient-Centered Medical Home: American Academy of Family Physicians (AAFP), American Academy of Pediatrics (AAP), American College of Physicians (ACP), and the American Osteopathic Association (AOA);2007.

31. Croghan T, Brown J. Integrating Mental Health Treatment into the Patient Centered Medical Home. (Prepared by Mathematica Policy Research under Contract No. HHSA290200900019I TO2.). Rockville, MD: Agency for Healthcare Research and Quality;2010.

32. Cope D, Sherman S, Robbins A. Restructuring VA ambulatory care and medical education: the PACE model of primary care. Acad Med. 1996;71(7):761.

33. Sherman S, Chapman A, Garcia D, Braslow J. Improving recognition of depression in primary care; a study of evidence-based quality improvement. Jt Comm J Qual Saf. 2004;30(2):80-88.

34. Rubenstein L, Meredith L, Parker L, et al. Impacts of evidence-based quality improvement on depression in primary care. J Gen Intern Med. 2006;21:1027-1035.

35. Gittell JH, Seidner R, Wimbush J. A relational model of how highperformance work systems work. Organizational Science. 2009:1-17.

36. McDonald K, Sundaram V, Bravata D, et al. Care Coordination. Technical Review 9 (Prepared by the Stanford University-UCSF Evidencebased Practice Center under contract 290020017). Rockville, MD: Agency for Healthcare Research and Quality;2007.
37. Gittell JH, Fairfield KM, Bierbaum B, et al. Impact of relational coordination on quality of care, postoperative pain and functioning, and length of stay: a nine-hospital study of surgical patients. Med Care. 2000;38(8):807-819.

38. Rubenstein L, Chaney E, Ober $\mathbf{S}$, et al. Using evidence-based quality improvement methods for translating depression collaborative care research into practice. Families, Systems, and Health. 2010;28:91-113.

39. Fortney J, Enderle M, McDougall S, et al. Implementation outcomes of evidence-based quality improvement for depression in VA community based outpatient clinics. Implement Sci. 2012;7:30.

40. Klein S. The Veterans Health Administration: Implementing PatientCentered Medical Homes in the Nation's Largest Integrated Delivery System: The Commonwealth Fund;2011.

41. VHA Handbook 1058.05: VHA operation activities that may constitute research. In:, Department of Veterans Affairs, ed. Washington, DC: Veterans Health Administration; 2011.

42. Root Cause Analysis in Health Care: Tools and Techniques, Fourth Edition: Joint Commission Resources; 2009.

43. McLaughlin CP, Kaluzny AD. Continuous Quality Improvement in Health Care. Sudbury: Jones \& Bartlett Publishers; 2005.

44. Pomerantz A, Shiner B, Watts B, et al. The White River model of colocated collaborative care. Families, Systems, and Health. 2010;28(2):114-129.

45. Pomerantz AS, Cole BH, Watts BV, Weeks WB. Improving efficiency and access to mental health care: Combining integrated care and advanced clinical access. General Hospital Psychiatry. 2008;30:546-551.

46. Stetler CB, Mittman BS, Francis J. Overview of the VA Quality Enhancement Research Initiative (QUERI) and QUERI theme articles: QUERI Series. Implement Sci. 2008;3:8.

47. Koenig CJ, Maguen S, Daley A, Cohen G, Seal KH. Passing the baton: a grounded practical theory of handoff communication between multidisciplinary providers in two Department of Veterans Affairs outpatient settings. J Gen Intern Med. 2013;28(1):41-50.

48. Pomerantz AS, Corson JA, Detzer MJ. The challenge of integrated care for mental health: leaving the 50 minute hour and other sacred things. J Clin Psychol Med settings. 2009;16(1):40-46.

49. Tew J, Klaus J, Oslin D. The Behavioral Health Laboratory: Building a stronger foundation for the patient-centered medical home. Families, Systems, and Health. 2010;28(2):130-145.

50. Pincus HA. The future of behavioral health and primary care: drowning in the mainstream or left on the bank? Psychosomatics. 2003;44(1):111.

51. Nutting PA, Gallagher KM, Riley K, White S, Dietrich AJ, Dickinson WP. Implementing a depression improvement intervention in five health care organizations: experience from the RESPECT-Depression trial. Adm Policy Ment Health. 2007;34(2):127-137.

52. Yano E, Fleming B. Canelo, et al. National Survey Results for the Primary Care Director Module of the VHA Clinical Practice Organizational Survey. Sepulveda: VA HSR\&D Center for the Study of Healthcare Provider Behavior; 2008.

53. Post E, Metzger M, Dumas P, Lehmann L. Integrating mental health into primary care within the Veterans Helath Administration. Families, Systems, and Health. 2010;28(2):83-90.

54. Pomerantz A, Sayers S. Primary care-mental health integration in healthcare in the Department of Veterans Affairs. Families, Systems, and Health. 2010;28(2):78-82. 